\title{
Video Article \\ Human Neural Organoids for Studying Brain Cancer and Neurodegenerative Diseases
}

\author{
Erika Cosset* ${ }^{{ }^{1}}$, Manon Locatelli* ${ }^{2}$, Antoine Marteyn $^{2}$, Pierre Lescuyer $^{3}$, Florence Dall Antonia ${ }^{3}$, Flavio Maurizio Mor ${ }^{4}$, Olivier Preynat-Seauve ${ }^{5}$, \\ Luc Stoppini ${ }^{4}$, Vannary Tieng ${ }^{2}$ \\ ${ }^{1}$ Laboratory of Tumor Immunology, Translational Research Center in Onco-Hematology, Department of Internal Medicine Specialties, Faculty of Medicine, University of \\ Geneva \\ ${ }^{2}$ Department of Pathology and Immunology, University Medical Center, University of Geneva \\ ${ }^{3}$ Laboratory of Toxicology and Therapeutic Drug Monitoring, Geneva University Hospitals \\ ${ }^{4}$ Tissue Engineering Laboratory, Hepia/HES-SO, University of Applied Sciences Western Switzerland \\ ${ }^{5}$ Laboratory of Experimental cell therapy, Department of Diagnostics, Geneva University Hospitals \\ "These authors contributed equally
}

Correspondence to: Erika Cosset at Erika.Cosset@unige.ch

URL: https://www.jove.com/video/59682

DOI: doi:10.3791/59682

Keywords: Bioengineering, Issue 148, neural organoid, glioblastoma multiforme, cancer stem cells, human embryonic stem cells, dopaminergic neurons, neurodegenerative disease

Date Published: 6/28/2019

Citation: Cosset, E., Locatelli, M., Marteyn, A., Lescuyer, P., Dall Antonia, F., Mor, F.M., Preynat-Seauve, O., Stoppini, L., Tieng, V. Human Neural Organoids for Studying Brain Cancer and Neurodegenerative Diseases. J. Vis. Exp. (148), e59682, doi:10.3791/59682 (2019).

\section{Abstract}

The lack of relevant in vitro neural models is an important obstacle on medical progress for neuropathologies. Establishment of relevant cellular models is crucial both to better understand the pathological mechanisms of these diseases and identify new therapeutic targets and strategies. To be pertinent, an in vitro model must reproduce the pathological features of a human disease. However, in the context of neurodegenerative disease, a relevant in vitro model should provide neural cell replacement as a valuable therapeutic opportunity.

Such a model would not only allow screening of therapeutic molecules but also can be used to optimize neural protocol differentiation [for example, in the context of transplantation in Parkinson's disease (PD)]. This study describes two in vitro protocols of 1) human glioblastoma development within a human neural organoids (NO) and 2) neuron dopaminergic (DA) differentiation generating a three-dimensional (3D) organoid. For this purpose, a well-standardized protocol was established that allows the production of size-calibrated neurospheres derived from human embryonic stem cell (hESC) differentiation. The first model can be used to reveal molecular and cellular events occurring during in glioblastoma development within the neural organoid, while the DA organoid not only represents a suitable source of DA neurons for cell therapy in Parkinson's disease but also can be used for drug testing.

\section{Video Link}

The video component of this article can be found at https://www.jove.com/video/59682/

\section{Introduction}

The World Health Organization (WHO) classifies astrocytomas as low grade (grade I to II) or high grade (grade III and IV). Glioblastoma multiforme (GBM) is an astrocytoma grade IV, the most lethal of primary brain tumors, that is resistant to all current forms of treatments ${ }^{1}$. Despite standard-of-care therapy including neurosurgery, chemotherapy, and radiotherapy, GBM remains fatal and the 15-month overall survival rate has not dramatically changed over the past 15 years ${ }^{2}$. To make significant progress in understanding GBM pathogenesis, the use of relevant models is key. So far, the study of GBM has relied on cell lines, rodent organotypic slices, and xenotransplantation of patient-derived cells into mice or transgenic mice developing spontaneous tumors ${ }^{3,4}$. Although these models have been useful to study brain metastasis and tumor aggressiveness, they are restricted by differences among species, and resulting conclusions may be incorrectly translated to human tissues. Moreover, existing models with human cells are also limited by the absence of host tissue/tumor interactions ${ }^{3,4}$. Experimental models are critical for the translation from basic science to therapeutic targets. Therefore, describing a protocol to produce in vitro human neural organoids cocultured with GBM-initiating cells (GICs) can provide a relevant system that mimics morphological and functional features of GBM development. This system reproduces some in vivo features of GBM developmentsuch as diffuse migration of invading cells and necrosis areas, and it highlights gene expression relevant to tumor biology. As previously revealed, some critical microRNAs are induced during GIC development within $3 \mathrm{D}$ nervous tissue ${ }^{5,6}$.

$\mathrm{PD}$ is a major neurodegenerative disorder and associated with the degeneration of multiple neuronal subtypes ${ }^{7}$. Even if a progressive onset of symptoms (e.g., bradykinesia, asymmetric rest tremor, rigidity and posture instability) characterizes the disease, its exact etiology is not clearly established. Indeed, many studies have highlighted evidence that major risk factors can result from a combination of genetic and environmental factors. Parkinsonian symptoms are associated with the bilateral degeneration of dopaminergic neurons in the substancia nigra (SN), leading 
to the disappearance of dopaminergic (DA) axons projecting to the striatum ${ }^{8,9}$. Therefore, the reduction of striatal dopamine levels is correlated with progression of motor dysfunction in PD patients. Dopaminergic neurons contain tyrosine hydroxylase (TH), a key enzyme in the synthesis of catecholaminergic neurotransmitters that converts the amino acid L-tyrosine to L-3,4-dihydroxyphenylalanine (L-DOPA, a dopamine precursor) to dopamine $^{10}$. Early loss of TH activity followed by a decline in TH protein expression is a hallmark of PD.

This study describes two protocols using human neural organoids, with one specifically oriented towards a midbrain-like phenotype enriched with TH-positive cells.

\section{Protocol}

This protocol follows the guidelines of University of Geneva's human research ethics committee.

\section{Maintenance and culture of undifferentiated human embryonic stem cells (hESCs)}

1. Perform maintenance and expansion of hSECs on feeder-free conditions by pre-coating dishes with a specific extracellular matrix.

1. Thaw $300 \mu \mathrm{L}$ of extracellular matrix at $4{ }^{\circ} \mathrm{C}$ (typical range concentration $18-22 \mathrm{mg} / \mathrm{mL}$, keep on ice) and gently mix with $15 \mathrm{~mL}$ of cold DMEM medium to avoid a premature gelation of the extracellular matrix. Add $7.5 \mathrm{~mL}$ of the extracellular matric to both T150 flasks.

2. Incubate the dishes coated with extracellular matrix at $37^{\circ} \mathrm{C}$ for at least $1 \mathrm{~h}$ (maximum overnight).

3. Remove the medium and seed hESCs to a density of $6.5 \times 10^{4} \mathrm{cell} / \mathrm{cm}^{2}$.

2. Maintain $\mathrm{H} 1$ (hESC cell line) in hESC medium and $1 \%$ penicillin/streptomycin.

3. Pass the cells with enzymatic procedure: add $7.5 \mathrm{~mL}$ of enzymatic solution to a $T 75 \mathrm{~cm}^{2}$ flask over a period of $1-2 \mathrm{~min}$ at $37{ }^{\circ} \mathrm{C}$. Once cells are completely detached, add $7.5 \mathrm{~mL}$ of DMEM-F12 then centrifuge for $5 \mathrm{~min}$ at $300 \mathrm{xg}$. To allow for better survival, re-plate cells at the desired density onto extracellular matrix-coated dishes, in the same medium containing Rho-associated protein kinase (ROCK) inhibitor (10 $\mu \mathrm{M})$ for $24 \mathrm{~h}$.

\section{2. hESC-derived neural organoids for GBM studies}

1. $24 \mathrm{~h}$ before starting the $3 \mathrm{D}$ culture, replace the $\mathrm{hESC}$ medium with a serum-free medium supplemented with $10 \mu \mathrm{M}$ ROCK inhibitor (both components are necessary to support cell survival and spontaneous neurosphere formation during the aggregation phase in a microwell plate). The cells should be at $60 \%$ confluency. The next day (day 0 ), detach $\mathrm{hESC}$ colonies as single cells: remove the medium, then rinse with PBS without $\mathrm{Ca}^{2+} / \mathrm{Mg}^{2+}$, add $5 \mathrm{~mL}$ of enzymatic dissolution solution, and incubate at $37^{\circ} \mathrm{C}$ for $1-2 \mathrm{~min}$.

2. Collect the cells in serum-free medium with $10 \mu \mathrm{M}$ of ROCK inhibitor and centrifuge the cells at $300 \times g$ for 5 min. Remove the supernatant and count the cells in $10 \mathrm{~mL}$ of serum-free medium supplemented with $10 \mu \mathrm{M}$ of ROCK inhibitor.

3. In parallel, rinse the microwell plate with $2 \mathrm{~mL}$ of serum-free medium per well and centrifuge the plate at $1200 \times \mathrm{g}$ for $5 \mathrm{~min}$ to remove all bubbles, which can prevent neurosphere formation.

4. Prepare $28.2 \times 10^{6}$ of cells in $12.5 \mathrm{~mL}$ of serum-free medium supplemented with $10 \mu \mathrm{M}$ ROCK inhibitor. Dispense 1000 cells/microwell. Centrifuge the cells at $300 \times g$ for 5 min and place the plate in the incubator at $37^{\circ} \mathrm{C}$ overnight (maximum $36 \mathrm{~h}$ ). For example: to obtain 30 human neural organoids, use one T150 flask at $70 \%-80 \%$ of confluence (about 30 million cells).

5. The next day (day 1), collect the spheres (with a P1000) and place them in a 6 well plate. In each well, add 2 mL of B27 medium and DMEMF12 GlutaMAX and Neurobasal medium (mix at 1:1), supplemented with $1 \%$ B27 supplements and $1 \%$ non-essential amino acids (NEAA). To promote fast neural induction, supplement the medium with dual-SMAD inhibition cocktail, composed of $10 \mu \mathrm{M}$ TGF $\beta / A c t i v i n / N o d a l$ inhibitor and $0.5 \mu \mathrm{M}$ bone morphogenic protein (BMP) inhibitor. From this step forward, the spheres are cultured in rotation (60 rpm, orbital shaker). The rotation is critical to prevent the spheres from sticking together or to the plate.

6. Change the medium every 2-3 days: bend the plate and let the spheres fall down for $5 \mathrm{~min}$, remove half of the medium ( $2 \mathrm{~mL}$ ), and add $2 \mathrm{~mL}$ of fresh B27 medium supplemented with growth factors and inhibitors. Do not centrifuge the spheres.

7. Perform neural induction according to the following time course:

1. From days 1-4, culture the spheres in B27 medium supplemented with dual-SMAD. The dual-SMAD inhibition cocktail (10 $\mu M$ TGF $\beta /$ Activin/Nodal inhibitor and $0.5 \mu \mathrm{M}$ BMP inhibitor) promote the neural induction.

2. From days 4-11, promote proliferation of hESC-derived neural rosettes (into the spheres), by adding $10 \mathrm{ng} / \mathrm{mL}$ epidermal growth factor (EGF) and $10 \mathrm{ng} / \mathrm{mL}$ basic fibroblast factor (bFGF) to the B27 medium supplemented with dual-SMAD cocktail.

NOTE: On day 11, most of the cells should be positive for Nestin.

3. From days 11-13, culture the spheres in B27 medium supplemented with $0.5 \mu \mathrm{M}$ BMP inhibitor.

4. From days 13-21, culture the spheres in B27 medium supplemented with $10 \mathrm{ng} / \mathrm{mL}$ glial derived neurotrophic factor (GDNF), $10 \mathrm{ng} / \mathrm{mL}$ brain derived neurotrophic factor (BDNF) and $1 \mu \mathrm{M}$ of $\mathrm{Y}$-secretase inhibitor. GDNF and BDNF promote neuronal and glial differentiation. The $\mathrm{y}$-secretase inhibitor allows for greater neural maturation.

5. On day 21, plate the spheres (about 1,000 spheres) on a hydrophilic polytetrafluoroethylene (PTFE) membrane $(6 \mathrm{~mm}$ diameter, 0.4 $\mu \mathrm{m}$ ) deposited on a culture plate insert designed for 6 well plate. Stop any rotation from this step. The presence of rosettes, observed with a bright-field microscope, indicate the initiation of neural differentiation. The neural rosettes can be observed 2-3 days after plating spheres on the PTFE membrane.

6. Add $1 \mathrm{~mL}$ of B27 medium supplemented with growth factors and inhibitors (as followed) to each well underneath the membrane insert, every 2-3 days (usually on Monday, Wednesday and Friday), for a following 3 weeks of differentiation.

7. From days 21-25, cultivate human neural organoids in the same neural maturation medium (Cf. step 2.7.4).

8. From days $25-28$, only complement B27 medium with $1 \mu \mathrm{M} y$-secretase inhibitor.

9. From days $28-39$, stop adding the $y$-secretase inhibitor and continue human neural organoid culture in B27 medium only. 
NOTE: After 3 weeks, neural organoids are ready to use for GIC implantation. Along the neural maturation, a decrease of neural immature marker Nestin and increase of mature neural markers $\beta 3$-tubulin and GFAP were observed.

\section{Isolation and cultivation of glioblastoma-initiating cells (GICs)}

1. Isolate GICs by fragmenting a high grade human GBM biopsy. Transfer the piece of tumor in a beaker containing $0.25 \%$ trypsin in $0.1 \mathrm{mM}$ EDTA (4:1) and slowly stir at $37^{\circ} \mathrm{C}$ for $30-60$ min (depending on tumor size).

2. Plate the dissociated cells in $75 \mathrm{~cm}^{2}$ tissue culture flasks plated at 2,500-5,000 cells per $\mathrm{cm}^{2}$ in GIC medium: DMEM/F-12 medium (1:1) containing $1 \% \mathrm{~N} 2,1 \% \mathrm{~B} 27$, and $1 \%$ G5 supplements (to favor GIC survival), supplemented with bFGF and EGF (both at $10 \mathrm{ng} / \mathrm{Ml}$, to promote stemness) and $1 \%$ of penicillin/streptomycin.

3. Once the GIC is well-established and growing, remove the N2 and G5 supplements from the GIC medium.

4. One day before adding the cells onto the organoid, dissociate the GICs and count them.

5. Rinse the microwell plate with $2 \mathrm{~mL}$ of GIC medium and centrifuge the plate at the maximum speed to remove bubbles $(1000 \times g)$. Place the GICs at 1,000 cells to obtain one gliomasphere per microwell. Incubate overnight at $37^{\circ} \mathrm{C}$ (Figure 1C). This step is key and allows for wellcalibrated GICs (an example of necrotic and over-sized GICs is shown in Figure 2A,C).

6. To initiate GBM invasion, add one gliomasphere on top of the neural tissue with a large bore pipet tip (Figure 1F). Carefully place the 6 well plate back in the incubator.

\section{4. hESC-derived dopaminergic organoids for PD studies}

1. Day 0: Amplify hESCs in 2D culture up to $60 \%$ confluency (day 0 ), then replace stem cell media used to maintain pluripotency features of hESCs with a serum-free medium. Start neural induction by supplementing culture medium with $0.5 \mu \mathrm{M}$ BMP inhibitor and $10 \mu \mathrm{M}$ TGF $\beta /$ Activin/Nodal inhibitor (dual-SMAD inhibition cocktail), then add $10 \mu \mathrm{M}$ ROCK inhibitor for $24 \mathrm{~h}$ to increase the survival rate of cells during passage.

2. Day 1: Prepare the microwell plate with $2.5 \mathrm{~mL}$ per well of serum-free medium supplemented with $0.5 \mu \mathrm{M}$ BMP inhibitor, $10 \mu \mathrm{M}$ TGF $\beta / A c t i v i n /$ Nodal inhibitor, and $10 \mu \mathrm{M}$ ROCK inhibitor. To specify cells towards the ventral pattern of the neural tube, add $100 \mathrm{ng} / \mathrm{mL}$ Sonic Hedgehog $(\mathrm{SHH}), 100 \mathrm{ng} / \mathrm{mL}$ fibroblast growth factor 8 (FGF8), and $2 \mu \mathrm{M}$ smoothened agonist. Centrifuge the plate (only with medium and without cells) at $1200 \times g$ for $5 \mathrm{~min}$ to remove air bubbles from the microwells.

1. After 1 day of neural induction in $2 \mathrm{D}$, remove the medium and quickly wash with PBS without $\mathrm{Ca}^{2+} / \mathrm{MgCl}^{2+}$. Dissociate the colonies in single cells suspension by adding $7.5 \mathrm{~mL}$ of recombinant enzymatic solution in a T75 $\mathrm{cm}^{2}$ flask. Incubate for $2 \mathrm{~min}$ at $37^{\circ} \mathrm{C}$ then complete with $7.5 \mathrm{~mL}$ of DMEM-F12.

2. Collect the cell suspension and centrifuge at $300 \times g$ for $5 \mathrm{~min}$. Remove the supernatant and count the cells in the same medium used to prepare the microwell plate.

3. Adjust the medium volume to obtain a cell suspension allowing to form neurospheres containing 1000 cells per microwell (for example, the microwell plate used here contains 4,700 microwells per well). So, prepare 4.7 million cells in $2.5 \mathrm{~mL}$ of medium and add it to the previous $2.5 \mathrm{~mL}$ of medium already placed in the plate.

4. In order to correctly distribute the cells in each microwell, gently shake the plate, and centrifuge the microwell plate $300 \times \mathrm{g}$ for $5 \mathrm{~min}$. Incubate the plate at $37^{\circ} \mathrm{C}$ for $24 \mathrm{~h}$ to generate spheres.

3. Day 2: Gently flush the microwells with the medium and collect then transfer the spheres in tissue-treated six-well plate. Replace medium with Neurobasal medium supplemented with $1 \%$ B27, 1x NEAA, 2 mM L-glutamine, and $1 \%$ of penicillin/ streptomycin. Additionally, add regionalization factors $\mathrm{SHH}$, FGF8, smoothened agonist, and dual-SMAD inhibition small molecules.

1. Place spheres in rotation at $37^{\circ} \mathrm{C}(60 \mathrm{rpm}$, orbital shaker $)$ and change half-medium freshly supplemented every $2-3$ days.

4. Day 3: To enhance neural induction and convert to neural progenitors with a midbrain identity, supplement the medium with $3 \mu \mathrm{M}$ GSK-3 $\beta$ inhibitor, which activates the Wnt/ $\beta$-catenin pathway. Maintain GSK-3 $\beta$ inhibitor in the medium up to day 13 . Split into two new tissue-treated 6 well plates to reduce both sphere density per well and avoid sphere aggregation.

NOTE: At Day 8, most of the cells should be positive for Nestin.

5. Day 8: Start the neural maturation: replace regionalization factors SHH, FGF8, smoothened agonist, and dual-SMAD inhibition cocktail with $0.5 \mathrm{mM}$ dibutyryl cAMP (to favor maturation), $20 \mathrm{nM}$ inhibitor of histone deacetylase (for cell cycle exit), $1 \mu \mathrm{M} \gamma$-secretase inhibitor and growth factors, $10 \mathrm{ng} / \mathrm{mL}$ GDNF, $10 \mathrm{ng} / \mathrm{mL}$ BDNF, $1 \mathrm{ng} / \mathrm{mL}$ transforming growth factor $\beta 3$ (TGF 33 ), and $5 \mathrm{ng} / \mathrm{mL}$ FGF20 (both favor DA progenitor survival). Change the medium every 2-3 days.

6. Day 21: Generate the neural organoid: seed around 100 neurospheres under air-liquid interface conditions on PTFE membrane (6 mm diameter). Transfer the membrane to a culture plate insert $(0.4 \mathrm{~mm})$ and add $1.2 \mathrm{~mL}$ of neural maturation medium used for neurosphere differentiation as previously described.

1. Stop any rotation from this step. Change the medium every 2-3 days until the required differentiation time point is achieved. NOTE: Regarding neural maturation, a decrease of the neural immature marker Nestin and increase of mature neural markers $\beta 3$-tubulin and GFAP were observed. High TH and NURR1 expressions were observed (Figure 3C) and confirm neural organoid maturity ${ }^{11}$.

\section{Quantification of TH and Nurr1 gene expression for validation of dopaminergic differentiation}

1. RNA extraction: On the indicated day of differentiation, lyse 40 neurospheres with $350 \mu \mathrm{L}$ of RLT buffer (provided in RNA extraction kit) supplemented with $3.5 \mu \mathrm{L}$ of 2-mercaptoethanol. Extract the RNA from the lysed neurospheres using an RNA extraction kit following the manufacturer's instructions. 
2. Quantify total RNA concentrations.

3. Perform reverse transcription of $300 \mathrm{ng}$ of the total RNA extraction using reverse transcription kit for quantitative real-time polymerase chain reaction ( $\mathrm{PPCR}$ ) and follow the manufacturer's instructions .

4. Perform qPCR analysis on real-time PCR detection systems, based on asymmetrical cyanine dye detection. Normalize the data with housekeeping genes: glyceraldehyde-3-phosphate dehydrogenase (GAPDH) and elongation factor 1-alpha (EF1). Sequences of primers are described in Table 1.

\section{High pressure liquid chromatography (HPLC) detection}

1. Use high pressure liquid chromatography (HPLC) with electrochemical detection to detect the presence of dopamine. Dopamine was extracted by lysing neural organoids in $100 \mathrm{~mL}$ of $0.1 \mathrm{~N}$ perchloric acid $(\mathrm{HClO} 4)$ for $15 \mathrm{~min}$ at $4{ }^{\circ} \mathrm{C}$ with a vigorous vortexing every 5 min. After centrifugation, collect and store the supernatant at $-20{ }^{\circ} \mathrm{C}$ for dopamine dosage.

2. Use a C-18 column $(5 \mu \mathrm{m}, 4.6 \mathrm{~mm} \times 150 \mathrm{~mm})$ to separate the analytes by reversed-phase HPLC in isocratic mode at a flow rate of $1 \mathrm{~mL} /$ minute. Detection of dopamine should be carried out using a coulometric detector with the conditioning cell set at a potential of $+200 \mathrm{mV}$.

\section{Raw data recording with microelectode array (MEA) platform}

1. Use a dissection microscope to transfer neurospheres to the center of a porous MEA device.

2. Use an amplifier and data acquisition system for electrophysiological recordings. Measure the signal-to-noise ratio (SNR) as the standard deviation of the voltage during a 5 min recording, using the signal as the average peak-to-peak voltage of the spikes recorded in the same 5 min periods.

\section{Representative Results}

The critical steps of this protocol must be well-identified and handled properly. Therefore, a diagram of culture conditions indicating the timelapse for each step as well as the compounds used for the differentiation protocol are illustrated in Figure 1A and Figure 3A for NO plus GBM and DA neural organoids, respectively. Figure 1B,C,D,E,F illustrates the cells, spheres, and NO and show the typical morphology for each step. Figure $1 \mathrm{G}, \mathrm{H}, \mathrm{I}$ illustrates immunofluorescence staining with some neural markers. 

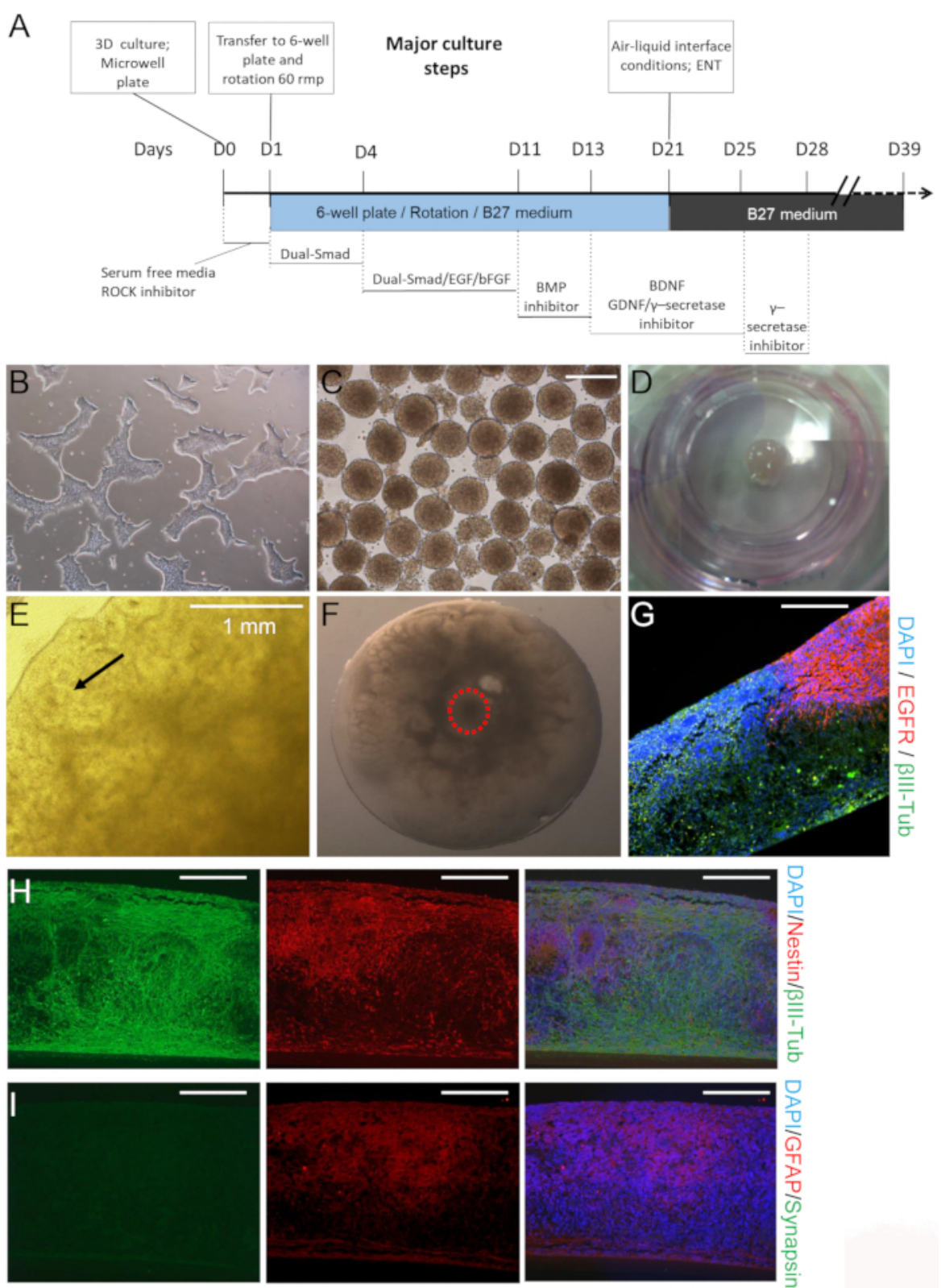

Figure 1: Human neural organoid (NO) differentiation protocol. (A) Standardized protocol for the generation of NO derived from human embryonic stem cells (hESC). (B) hESCs are maintained on extracellular matrix in hESC medium. (C) Microwell plates were used to generate calibrated neurospheres. At 2 weeks, neurospheres were plated onto the insert containing a PTFE membrane $(\mathrm{scale}$ bar $=50 \mu \mathrm{m}) .(\mathbf{D})$ Macroscopic view of NO into the insert in one well of a 6 well plate. During the first days, rosettes were observed (black arrow) (E). (F)

Macroscopic view of a NO plus GIC sphere on the top. (G-I) Immunofluorescence analysis of NO plus GIC sphere (EGFR-positive; scale bar = $50 \mu \mathrm{m})(\mathbf{G})$ and NO alone, which showed immune reactivity for the neuronal marker ßlll-tubulin and slightly positive for nestin; however, synapsin 1 showed a weak signal $(\mathbf{H}, \mathbf{I})$ (scale bars $=100 \mu \mathrm{m}$ and $50 \mu \mathrm{m}$, respectively). Please click here to view a larger version of this figure. 

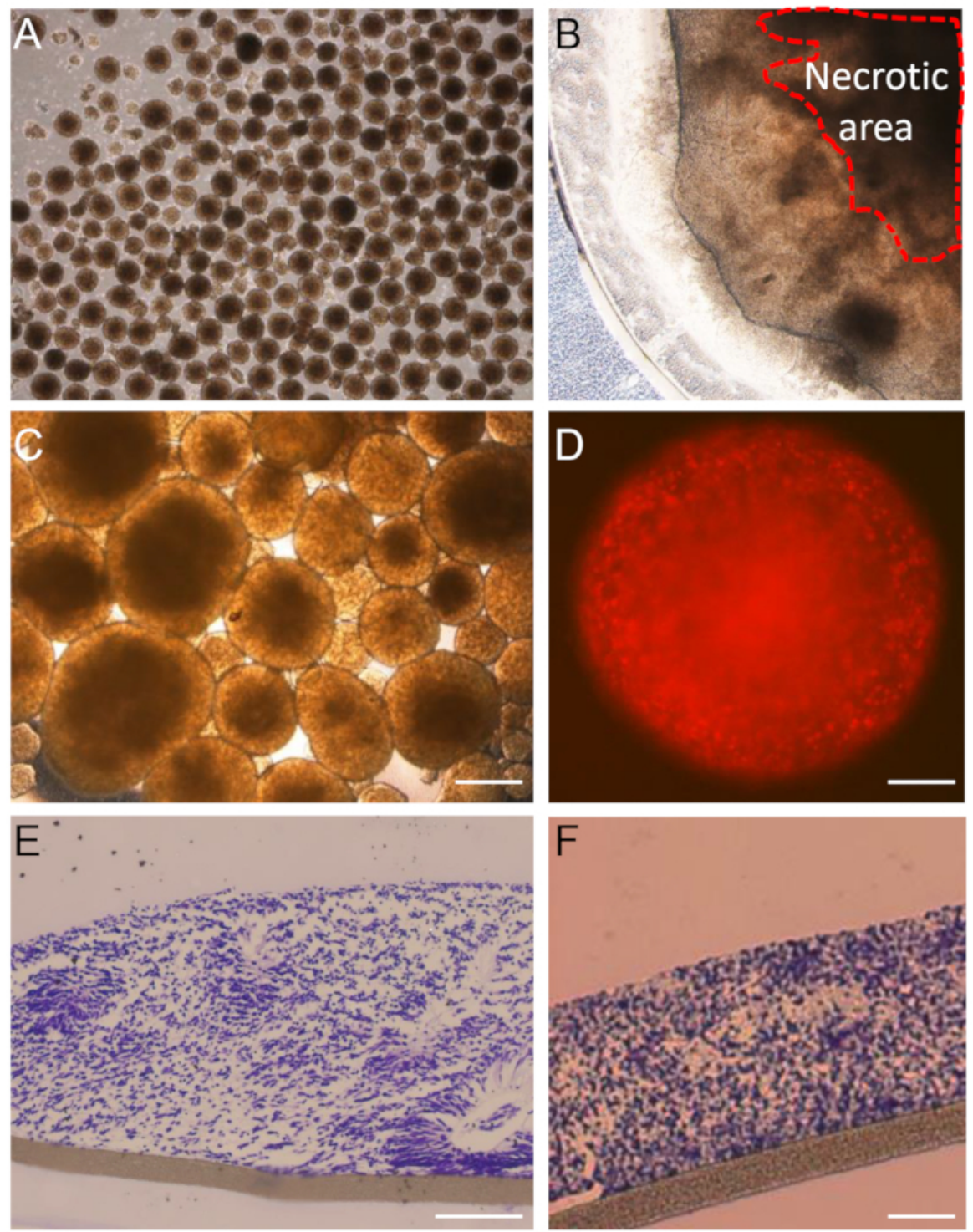

Figure 2: Illustration of necrotic spheres and immature NO. The neurospheres (A) and NO (B) can undergo necrosis when they are too numerous in the well or oversized $(\mathbf{C})$ (scale bar $=10 \mu \mathrm{m}$ ). (D) One GIC infected with a tomato reporter help to track tumor cell invasion in NO, scale bar, $10 \mu \mathrm{m}$. Example of immature NO with neural tubes $(\mathbf{E})$ and no neural tubes $(\mathbf{F})$ (scale bar $=50 \mu \mathrm{m})$. Please click here to view a larger version of this figure. 


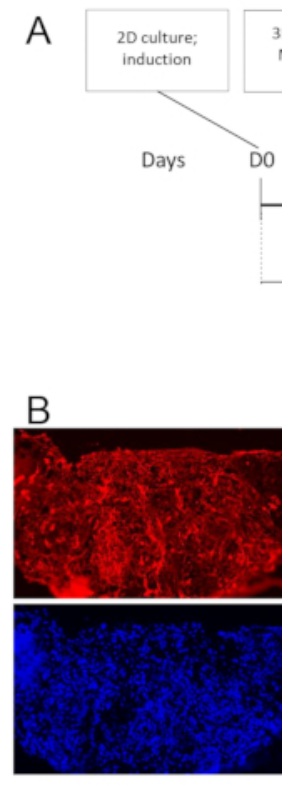

D

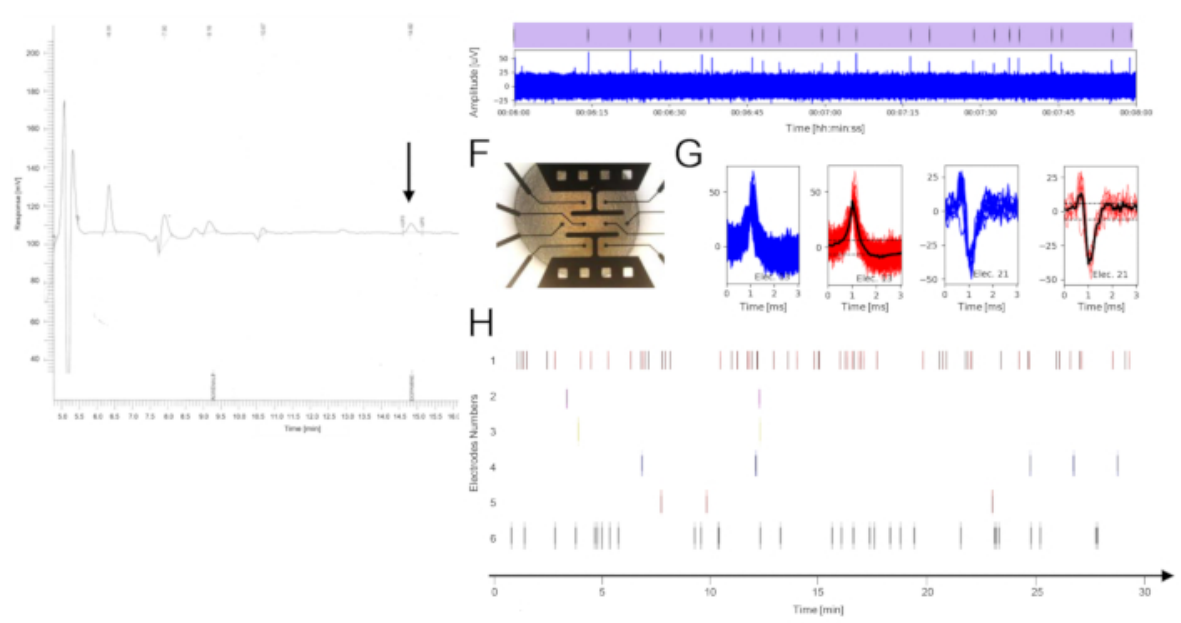

Figure 3: Standardized protocol for generation of DA neural organoid and electrophysiological and morphological analysis. (A) Standardized protocol for the generation of DA neural organoids. (B) Immunofluorescence analysis of DA neural organoid; TH-immunoreactive cells co-expressing Nurr1, a midbrain specific marker (scale bar $=50 \mu \mathrm{m})$. Data are represented as mean \pm SEM $(n=3)$. (C) Graphs represent kinetics of TH and Nurr1 gene expression evaluated by qRT-PCR. (D) Representative HPLC: dopamine peak (arrow) was detected by HPLC from DA neural organoid lysate. (E) Example of raw data recorded with MEA platform. Each spike is displayed by a vertical line (time stamps), whereas the remaining trace is noise. (F) Picture representing a neurosphere deposited on the MEA. (G) Superposition of typical spikes (blue and red curves) detected from the raw data. The black bold curve indicates the average of the corresponding red curves. $(\mathbf{H})$ Raster plot showing the time stamps associated with each spike detected. The different colors highlight the different electrodes. Please click here to view a larger version of this figure.

\begin{tabular}{|l|l|l|}
\hline Gene & Foward & Reverse \\
\hline Nurr1 & GGCTGAAGCCATGCCTTGT & GTGAGGTCCATGCTAAACTTGACA \\
\hline TH & GCACCTTCGCGCAGTTCT & CCCGAACTCCACCGTGAA \\
\hline EEF1 & AGCAAAAATGACCCACCAATG & GCCTGGATGGTTCAGGATA \\
\hline GAPDH & GCACAAGAGGAAAGAGAGAGAACC & AGGGGAGATTCAGTGTGGGT \\
\hline
\end{tabular}

Table 1: Primers used in this protocol.

\section{Discussion}

One of the most critical aspects of this protocol includes the maintenance of hESC pluripotency during cell culturing and close monitoring of the spheres and neural organoid morphology. hESCs are very sensitive, and every manipulation can lead to early uncontrolled differentiation 
as well as cell death. In order to increase experimental reproducibility and avoid the occurrence of abnormal karyotype events, it is advised to cryopreserve several batches of hESCs at the lowest passage after validation of their chromosome stability. Moreover, it is recommended to thaw a new vial for each experiment and check the behavior of the cells every day. If the spheres are less refractive with abnormal higher size, they will likely start to aggregate and die.

One improvement upon this system is either perfusion or implementing a vascularized system (by adding endothelial cells or within a 3D fluidic microchip $)^{12,13}$. However, controlling the thickness of the neural organoid $(\leq 300 \mu \mathrm{m})$ allows efficient passive perfusion of oxygen and nutriments and prevents necrosis. Another improvement is the introduction of immune cells (microglia). With these limitations in mind, neural organoids plus a GIC system may be a relevant tool for several reasons. First, this system allows drug screening to monitor how a therapeutic compound may affect an organoid or tumor cell. Second, cell-to-cell interactions can be studied, and micro-environmental determinants underlying individual and collective invasions can be visualized and explored ${ }^{5,6,13}$.

In the context of Parkinson's disease, a neural organoid enriched in DA neurons can represent a relevant and accurate 3D model to study disease development. In previous studies, Parkinson's patient-derived induced pluripotent stem cells differentiated towards DA neurons have been used to study the affected neuronal subtypes. Of note, some disease-related phenotypes such as the accumulation of $\alpha$-synuclein and sensitivity to oxidative stress have been observed ${ }^{14,15}$. Moreover, the neural organoid may be used as a tool to screen therapeutic molecules. However, specific and relevant readouts should be set up to evaluate DA neuron survival and functionality, such as dopamine production and electrophysiological activity. Altogether, this protocol provides two standardized and accurate stem cell-based approaches to generate neural organoids.

\section{Disclosures}

The authors declare no competing financial interests.

\section{Acknowledgments}

The authors thank la Ligue Genevoise Contre le Cancer (Geneva, Switzerland), ISREC Foundation (Lausanne, Switzerland), and the Clayton Foundation for Research (Houston, TX, USA) for financial support. Moreover, the authors thank HES-HO and the Wyss Center for financial support. We thank the Krause's lab for helpful discussions and support and Dr. Halah Kutaish for proofreading.

\section{References}

1. Louis, D. N. et al. The 2016 World Health Organization Classification of Tumors of the Central Nervous System: a summary. Acta Neuropathologica. 131 (6), 803-820 (2016).

2. Stupp, R. et al. Radiotherapy plus concomitant and adjuvant temozolomide for glioblastoma. New England Journal of Medicine. 352 (10), 987-996 (2005).

3. Daphu, I. et al. In vivo animal models for studying brain metastasis: value and limitations. Clinical and Experimental Metastasis. 30 (5), 695-710 (2013).

4. Huszthy, P. C. et al. In vivo models of primary brain tumors: pitfalls and perspectives. Neuro Oncology. 14 (8), $979-993$ (2012).

5. Nayernia, Z. et al. The relationship between brain tumor cell invasion of engineered neural tissues and in vivo features of glioblastoma. Biomaterials. 34 (33), 8279-8290 (2013).

6. Cosset, E. et al. Human tissue engineering allows the identification of active miRNA regulators of glioblastoma aggressiveness. Biomaterials. 107, 74-87 (2016).

7. Pringsheim, T., Jette, N., Frolkis, A., Steeves, T. D. The prevalence of Parkinson's's disease: a systematic review and meta-analysis. Movement Disorders. 29 (13), 1583-1590 (2014).

8. Rajput, A. H. et al. Globus pallidus dopamine and Parkinson's motor subtypes: clinical and brain biochemical correlation. Neurology. 70 (16 Pt 2), 1403-1410 (2008).

9. Jellinger, K. A. Pathology of Parkinson's's disease. Changes other than the nigrostriatal pathway. Molecular and Chemical Neuropathology. 14 (3), 153-197 (1991).

10. Bernheimer, H., Birkmayer, W., Hornykiewicz, O., Jellinger, K., Seitelberger, F. Brain dopamine and the syndromes of Parkinson's and Huntington. Clinical, morphological and neurochemical correlations. Journal of Neurological Science. 20 (4), 415-455 (1973).

11. Tieng, V. et al. Engineering of midbrain organoids containing long-lived dopaminergic neurons. Stem Cells and Development. 23 (13), 1535-1547 (2014).

12. Chen, C. S. 3D Biomimetic Cultures: The Next Platform for Cell Biology. Trends in Cell Biology. 26 (11), 798-800 (2016).

13. Friedl, P., Sahai, E., Weiss, S., Yamada, K. M. New dimensions in cell migration. Nature Reviews Molecular Cell Biology. 13 (11), $743-747$ (2012).

14. Byers, B. et al. SNCA triplication Parkinson's's patient's iPSC-derived DA neurons accumulate alpha-synuclein and are susceptible to oxidative stress. PLoS ONE. 6 (11), e26159 (2011).

15. Flierl, A. et al. Higher vulnerability and stress sensitivity of neuronal precursor cells carrying an alpha-synuclein gene triplication. PLoS ONE. 9 (11), e112413 (2014). 